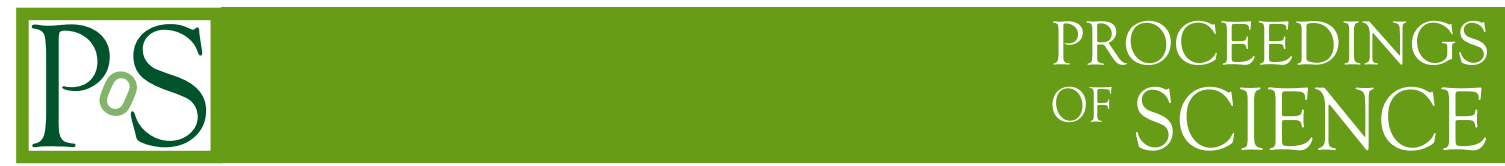

\title{
Kaon physics from lattice QCD
}

\author{
Vittorio Lubicz* \\ Università Roma Tre and INFN sezione di Roma Tre \\ E-mail: lubicz@fis.uniroma3.it
}

I review lattice calculations and results for hadronic parameters relevant for kaon physics, in particular the vector form factor $f_{+}(0)$ of semileptonic kaon decays, the ratio $f_{K} / f_{\pi}$ of leptonic decay constants and the kaon bag parameter $B_{K}$. For each lattice calculation a colour code rating is assigned, by following a procedure which is being proposed by the Flavianet Lattice Averaging Group (FLAG), and the following final averages are obtained: $f_{+}(0)=0.962(3)(4), f_{K} / f_{\pi}=$ $1.196(1)(10)$ and $\hat{B}_{K}=0.731(7)(35)$. In the last part of the talk, the present status of lattice studies of non-leptonic $K \rightarrow \pi \pi$ decays is also briefly summarized.

The XXVII International Symposium on Lattice Field Theory - LAT2009

July 26-31 2009

Peking University, Beijing, China

${ }^{*}$ Speaker. 


\begin{tabular}{cccccc} 
Collaboration & Quark action & $N_{f}$ & $a[\mathrm{fm}]$ & $\begin{array}{c}m_{\pi}^{\min } \\
{[\mathrm{MeV}]}\end{array}$ & $\begin{array}{c}\text { Kaon } \\
\text { observables }\end{array}$ \\
\hline $\begin{array}{c}\text { MILC } \\
\text { [+ FNAL, HPQCD, ... }]\end{array}$ & $\begin{array}{c}\text { Improved } \\
\text { Staggered }\end{array}$ & $2+1$ & $\geq 0.045$ & 230 & $f_{K}, B_{K}$ \\
PACS-CS & Clover (NP) & $2+1$ & 0.09 & 156 & $f_{K}$ \\
RBC/UKQCD & DWF & $2+1$ & $\geq 0.08$ & 295 & $\begin{array}{c}f_{+}(0), f_{K}, B_{K}, \\
K \rightarrow \pi \pi\end{array}$ \\
BMW & $\begin{array}{c}\text { Clover } \\
\text { Smeared }\end{array}$ & $2+1$ & $\geq 0.07$ & 190 & $f_{K}$ \\
JLQCD/TWQCD & Overlap & $\begin{array}{c}2 \\
{[2+1]}\end{array}$ & 0.12 & 290 & $B_{K}$ \\
ETMC & $\begin{array}{c}\text { Twisted } \\
\text { Mass }\end{array}$ & $\begin{array}{c}2 \\
{[2+1+1]}\end{array}$ & $\geq 0.07$ & 260 & $f_{+}(0), f_{K}, B_{K}$ \\
QCDSF & Clover (NP) & 2 & $\geq 0.07$ & 340 & $f_{K}$ \\
\hline
\end{tabular}

Table 1: Details of the unquenched lattice simulations which have been used for the studies of kaon physics. The relevant kaon observables computed in these calculations are listed in the last column.

\section{Introduction}

Kaon physics has always played a crucial role for our understanding of fundamental interactions in the flavour sector. Together with B-physics, for which however precise experimental information has become available only in the last decade, the study of kaon physics has allowed fundamental tests of the Standard Model and provides, at present, precise constraints on its possible new physics extensions.

While there are few selected processes in kaon physics, like the rare $K \rightarrow \pi v \bar{v}$ decays, which can be studied with almost negligible theoretical uncertainties, in most of the cases the extraction of the physical results also relies on our capability of controlling the non-perturbative effects of the strong interactions and, therefore, on the accuracy of lattice QCD calculations. This is the case, for example, of the determination of the CKM matrix element $V_{u s}$ from the study of semileptonic and leptonic kaon decays, or the theoretical prediction of the $\varepsilon_{K}$ parameter which controls the amount of indirect $\mathrm{CP}$ violation in $K^{0}-\bar{K}^{0}$ mixing. The hadronic parameters entering these processes, namely the vector form factor $f_{+}(0)$ of semileptonic kaon decays, the leptonic decay constant $f_{K}$ and the kaon bag parameter $B_{K}$, are the quantities to be computed in lattice QCD calculations.

In the last few years, the accuracy of the lattice predictions for kaon physics observables is significantly increased. Extensive unquenched lattice simulations have been performed by various lattice collaborations, using different lattice methods (i.e. different actions, renormalization techniques, etc.). A list of these simulations, their main details and a compilation of kaon observables which have been studied with them, is presented in table 1 . These simulations typically involve ensembles with different lattice spacings, which allow the continuum extrapolation to be eventually performed. They also include a number of relatively light simulated quark masses, with the 
lightest pion masses now typically smaller than $300 \mathrm{MeV}$. In this mass region, a controlled chiral extrapolation to the physical light quark masses can be performed, using in most of the cases chiral perturbation theory (ChPT) as a useful guideline for the extrapolation.

The relative abundance of unquenched lattice calculations, in which most (if not all) the systematic uncertainties are kept well under control and which use different approaches, characterized by different systematics, has allowed to reach in the determination of the corresponding hadronic parameters a significantly improved accuracy. It should be also noted that a table similar to table 1 for lattice studies of B-physics would present, today, a much shorter list.

In this talk I will mainly concentrate on reviewing the recent lattice results for the three hadronic parameters which are of particular interest for kaon physics, namely the vector form factor $f_{+}(0)$ of semileptonic kaon decays, the ratio $f_{K} / f_{\pi}$ of leptonic decay constants and the kaon bag parameter $B_{K}$. I will also mostly rely, for this task, on the detailed work which is being performed by the FLAG group, that will be introduced in the next section. For each lattice calculation, a colour code rating in the FLAG style will be assigned, and I will also present my best averages for the hadronic parameters. I will conclude this talk by briefly summarizing the status of lattice studies of non-leptonic $K \rightarrow \pi \pi$ decays.

\section{The FLAG working group: rating and averaging criteria for lattice results}

The improved control of systematic uncertainties achieved in the last few years by lattice QCD calculations, particularly for observables in the kaon sector, simplifies the task of deriving the corresponding lattice averages to be used in phenomenological analysis. This kind of task is one of those which is currently being addressed by the Flavianet Lattice Averaging Group (FLAG) [1], a working group of the Flavianet European network constituted in November 2007.

The aim of FLAG is to provide for each considered quantity, to the network's working groups and to the wider community, the following information: i) a collection of current lattice results and references; ii) a summary of the essential aspects of each calculation; iii) averages of lattice results.

The quantities which are being considered in the first FLAG report [1] are the light quark masses $\left(m_{u}, m_{d}, m_{s}\right)$, the $\mathrm{SU}(2)$ and $\mathrm{SU}(3)$ low energy constants, the kaon semileptonic form factor $f_{+}(0)$, the ratio of leptonic decay constants $f_{K} / f_{\pi}$ and the bag parameter $B_{K}$. It is clear from this list that a significant overlap exists between the FLAG work and the task I have been given at this conference. I will then take advantage of this overlap, and I will use for this talk several FLAG results. I am indebted and grateful for that to my colleagues in the FLAG group.

One of the FLAG proposals that I'm going to follow in this review concerns the way of summarizing the essential aspects of each lattice calculation. This is done by using an easy-to-read "colour code" classification. Specifically, a number of sources of systematic errors are identified and a colour with respect to each of these is assigned to each calculation. The prescription is as follows:

$\star$ when the systematic error has been estimated in a satisfactory manner and convincingly shown to be under control;

- when a reasonable attempt at estimating the systematic error has been made, although this could be improved;

when no or a clearly unsatisfactory attempt at estimating the systematic error has been made. 
It should be clear that the precise criteria used in determining the colour coding are unavoidably time-dependent. The sources of systematic error and definitions which are currently adopted by FLAG, and which I'm also going to follow in this talk, are:

- Chiral extrapolation:
$\star M_{\pi, \min }<250 \mathrm{MeV}$
- $250 \mathrm{MeV} \leq M_{\pi, \min } \leq 400 \mathrm{MeV}$
$M_{\pi, \min }>400 \mathrm{MeV}$

It is assumed that the chiral extrapolation is done with at least a three-point analysis. In case of nondegeneracies among the different pion states $M_{\pi}$ stands for an average pion mass.

- Continuum extrapolation:

$\star \quad 3$ or more lattice spacings, at least 2 points below $0.1 \mathrm{fm}$

- 2 or more lattice spacings, at least 1 point below $0.1 \mathrm{fm}$

otherwise

It is assumed that the action is $O(a)$-improved. The colour coding criteria for non-improved actions change as follows: one lattice spacing more needed.

- Finite-volume effects:

$\star \quad\left(M_{\pi} L\right)_{\min }>4$ or at least 3 volumes

- $\left(M_{\pi} L\right)_{\min }>3$ and at least 2 volumes

otherwise

It is assumed that $L_{\min } \geq 2 \mathrm{fm}$, otherwise a red dot will be assigned. In case of nondegeneracies among the different pion states $M_{\pi}$ stands for an average pion mass.

- Renormalization (where applicable):

$\star$ non-perturbative

- 2-loop perturbation theory (with a converging series)

otherwise

- Running (where applicable):

$\star$ non-perturbative

- otherwise

-

Of course any colour coding has to be treated with caution and it goes without saying that these criteria are subjective and evolving. Moreover, the extent to which each source of systematic uncertainty affects the lattice calculation is observable dependent. FLAG believes, however, that this attempt to introduce quality measures for lattice results, in spite of being necessarily schematic, will prove to be a useful guide.

The other main purpose of FLAG is to provide averages of lattice results. The average should only include, as far as possible, only "good quality" lattice calculations. This is implemented, in practice, by relying on the colour coding: unless special reasons are given for making an exception, the averages are restricted to data for which the colour code does not contain any red dot. In deriving the averages quoted in this talk, I will follow the same criterium.

There are two other criteria adopted by FLAG for computing the averages which, however, I'm not going to apply for the purposes of the present review. One is related to the publication status, for which a coloured symbol is also introduced: 
- Publication status:
A published or plain update of published results
$\mathbb{P}$ preprint
C conference contribution

The FLAG policy is to consider in the averages only calculation which have been published, i.e. which have been endorsed by a referee. While I find this policy perfectly justified for the FLAG purposes, I also believe that the same criterium is not suitable for the reviewer at the lattice conference. The latter is asked to concentrate the attention mainly on the new (and typically unpublished) results presented at the conference. It is the task of the reviewer, rather than of an external referee in this case, to judge the quality and the reliability of the presented results.

The other criterium adopted by FLAG is related to the number of flavours of dynamical quarks introduced in the simulation. The policy that is being followed by FLAG consists in presenting separate averages for the $N_{f}=2$ and $N_{f}=2+1$ calculations. This issue has been quite debated within the working group, and I personally do not consider the choice currently pursued by FLAG an optimal one for a review of lattice results. There are mainly three reasons for that, in my opinion, that I would like to mention here, since the issue is also relevant for the present review.

i) I believe that it would be useful to present, to the wider community of particle physics, the "best lattice result" in terms of just a single number, rather than two. When separate averages for the $N_{f}=2$ and $N_{f}=2+1$ results are quoted, the natural interpretation of the latter as the best result may not always correspond to the actual situation. The error due to the quenching of the strange quark is rather small in most of the cases, whereas other sources of systematic uncertainty could be better under control in the $N_{f}=2$ determination.

ii) There are cases, like the one of the semileptonic form factor discussed in the next section, in which the error due to the quenching of the strange quark in the $N_{f}=2$ calculation is evaluated and included in the systematic uncertainty. In other cases, the comparison between the $N_{f}=2$ and $N_{f}=2+1$ results shows, a posteriori, that the systematic effect due to the quenching of the strange quark is not visible within the currently reached accuracy (to the best of my knowledge, this is actually the case of all lattice calculations performed so far). In all these cases, I do not see any valid reason for ignoring the information coming from the $N_{f}=2$ calculations and for not combining together the two sets of results.

iii) The $N_{f}=2+1$ theory is not really "full QCD". Indeed, $N_{f}=2+1+1$ lattice calculations are already being performed (see for instance [2]). I would find unreasonable to simply forget the $N_{f}=2+1$ calculations when $N_{f}=2+1+1$ results will be available. For all those quantities for which the error due to the quenching of the charm quark will turn out (a posteriori) to be negligible, I will suggest again to average together $N_{f}=2+1$ and $N_{f}=2+1+1$ results.

For these reasons, in deriving lattice averages for the present review, I'm not going to follow the FLAG criterium as far as the number of dynamical flavours is concerned, and I will rather address this issue on a case by case basis.

\section{3. $\left|\mathbf{V}_{\mathrm{us}}\right|$ from semileptonic and leptonic kaon decays}

The most accurate determinations of the Cabibbo angle, or equivalently the CKM matrix element $V_{u s}$, come from the study of semileptonic $K \rightarrow \pi \ell v\left(K_{\ell 3}\right)$ and leptonic $K \rightarrow \ell v\left(K_{\ell 2}\right)$ decays. 
Very precise experimental measurements of the $K_{\ell 3}$ and of the ratio of $K_{\ell 2}$ over $\pi_{\ell 2}$ decay rates allow to determine the following combinations of CKM and hadronic parameters [3],

$$
\left|V_{u s}\right| f_{+}(0)=0.21664(48) \quad, \quad\left|\frac{V_{u s}}{V_{u d}}\right| \frac{f_{K}}{f_{\pi}}=0.27599(59),
$$

with an accuracy of about $2 \%$. In eq. (3.1), $f_{+}(0)$ is by convention the form factor for the $K^{0} \rightarrow$ $\pi^{-}$matrix element. Moreover, both $f_{+}(0)$ and the ratio of decay constants $f_{K} / f_{\pi}$ in eq. (3.1) are defined in the isospin symmetric limit $m_{u}=m_{d}$ (keeping the kaon and pion masses to their physical value) and neglecting electromagnetic corrections. These are therefore the quantities that are directly determined in lattice QCD simulations.

A determination of the hadronic parameters $f_{+}(0)$ and $f_{K} / f_{\pi}$, which assumes the validity of the Standard Model and it is independent of lattice QCD calculations, has been provided by FLAG [1]. It makes use of the first row unitarity constraint on the CKM matrix,

$$
\left|V_{u d}\right|^{2}+\left|V_{u s}\right|^{2}+\left|V_{u b}\right|^{2}=1
$$

which holds in the Standard Model. Since within present uncertainties the contribution of $\left|V_{u b}\right|$ in eq. (3.2) is numerically negligible, the unitarity constraint (3.2) can be combined with the two experimental results in (3.1) to provide a set of three equations and four unknowns: $\left|V_{u d}\right|,\left|V_{u s}\right|$, $f_{+}(0)$ and $f_{K} / f_{\pi}$. A precise determination of $\left|V_{u d}\right|$ is provided by the study of nuclear $\beta$-decays [4], based on the analysis of 20 different superallowed transitions:

$$
\left|V_{u d}\right|=0.97425(22)
$$

Using this result, one can then obtain a determination of the other three parameters,

$$
\begin{gathered}
\left|V_{u s}\right|=\left(1-\left|V_{u d}\right|^{2}\right)^{1 / 2}=0.22547(95) \\
f_{+}(0)=0.9608(46) \quad, \quad \frac{f_{K}}{f_{\pi}}=1.1925(56) .
\end{gathered}
$$

The estimates (3.5) of the hadronic parameters are benchmarks for the lattice results reviewed in this talk. Since both $f_{+}(0)$ and $f_{K} / f_{\pi}$ are equal to 1 in the $\mathrm{SU}(3)$-symmetric limit, what it is actually measured on the lattice are the $\mathrm{SU}(3)$ breaking effects, i.e. the differences $f_{+}(0)-1$ and $f_{K} / f_{\pi}-1$. Eq. (3.5) shows that, in order to provide a significant test of the Standard Model, these differences must be determined on the lattice with an accuracy of about $10 \%$ and $3 \%$ respectively. Of course, when exploiting new physics scenarios beyond the Standard Model, the unitarity relation (3.2) should not be assumed and the determinations in eq (3.5) are no longer valid.

\subsection{Semileptonic kaon decays: $\mathbf{f}_{+}(\mathbf{0})$}

The lattice determinations of $f_{+}(0)$ and, in the next section, of $f_{K} / f_{\pi}$, are now reviewed.

The standard approach to study the vector form factor of $K_{\ell 3}$ decays is based on SU(3) ChPT. In this framework, the vector form factor at zero momentum transfer has an expansion of the form $f_{+}(0)=1+f_{2}+f_{4}+\ldots$, where $f_{n}=O\left[m_{K, \pi}^{n} /\left(4 \pi f_{\pi}\right)^{n}\right]$ and the first term of the expansion is equal to 1 due to the current conservation in the SU(3)-symmetric limit. The Ademollo-Gatto 
theorem [5] shows that the deviation of $f_{+}(0)$ from 1 is at least quadratic in the breaking of SU(3). Moreover, the first correction $f_{2}$ receives contribution only from chiral loops and can be computed unambiguously in terms of the kaon and pion masses and the pion decay constant. It takes the value $f_{2}=-0.0226$ [6]. The problem of estimating $f_{+}(0)$ can be thus re-expressed as the problem of finding a prediction for $\Delta f=f_{+}(0)-\left(1+f_{2}\right)$. The reference estimate for this quantity is still the one obtained by Leutwyler and Roos in 1984 [7], using a general parameterization of the SU(3) breaking structure of the pseudoscalar meson wave functions. It reads $\Delta f=-0.016(8)$, which implies $f_{+}(0)=0.961(8)$.

Lattice QCD studies of $K_{\ell 3}$ decays started only relatively recently. The strategy, which allows to reach the required percent accuracy in the determination of the vector form factor, has been developed in ref. [8], and it is based on the calculation of the scalar form factor at maximum momentum transfer $\left(q_{\max }^{2}=\left(m_{K}-m_{\pi}\right)^{2}\right)$ through the ratio

$$
\frac{\left\langle\pi\left|\bar{s} \gamma_{0} u\right| K\right\rangle\left\langle\pi\left|\bar{s} \gamma_{0} u\right| K\right\rangle}{\left\langle\pi\left|\bar{u} \gamma_{0} u\right| \pi\right\rangle\left\langle K\left|\bar{s} \gamma_{0} s\right| K\right\rangle}=\frac{\left(m_{K}+m_{\pi}\right)^{2}}{4 m_{K} m_{\pi}} f_{0}\left(q_{\max }^{2}, m_{K}^{2}, m_{\pi}^{2}\right),
$$

where the external states are of pion and kaon at rest. The double ratio on the 1.h.s. of eq. (3.6) is equal to 1 in the $\mathrm{SU}(3)$ symmetric limit, and it can be evaluated on the lattice with sub-percent accuracy at the simulated values of pion and kaon masses. The physical form factor at zero momentum transfer, $f_{+}(0)=f_{0}(0)$, is then obtained by extrapolating $f_{0}\left(q_{\max }^{2}, m_{K}^{2}, m_{\pi}^{2}\right)$ to $q^{2}=0$ and to the physical meson masses.

A list of lattice results for $f_{+}(0)$ is collected in table 2, with the colour code in the FLAG style assigned for each calculation. The relevant simulation parameters for these calculations are given in table 3. The first lattice calculation of the form factor, by the $S P Q_{C D} R$ collaboration [8], was performed in the quenched approximation and with rather large values of simulated pion masses, $m_{\pi} \gtrsim 500 \mathrm{MeV}$. It gave the result $f_{+}(0)=0.960(5)(7)$, in remarkable agreement with the quark model prediction by Leutwyler and Roos [7]. Subsequently, unquenched calculations [11, 12, 13], performed with $N_{f}=2$ dynamical fermions but still large values of simulated pion masses, confirmed the quenched result. The first lattice calculation of $f_{+}(0)$ which aims to keep under control all sources of systematic uncertainties has been performed by the RBC/UKQCD collaboration [9]. This year, a second calculation of comparable accuracy has been presented by ETMC [10]. Both the update of the RBC/UKQCD 07 result and the new ETMC 09 calculation have been discussed in the parallel session at this conference $[14,15]$.

The RBC/UKQCD calculation [9] uses the DWF action with $N_{f}=2+1$ dynamical quarks, and pion masses as light as $330 \mathrm{MeV}$. The colour code rating for this calculation, displayed in table 2, includes a red square for the continuum extrapolation since the simulation has been performed at a single value of the lattice spacing $(a \simeq 0.11 \mathrm{fm})$. A $4 \%$ of systematic error on $\left(1-f_{+}\right)$due to discretization effects is assigned on the basis of the parametric estimate $\sigma_{\text {discr }} \sim\left(a \Lambda_{Q C D}\right)^{2}$, assuming $\Lambda_{Q C D} \simeq 300 \mathrm{MeV}$. In the parallel talk at this conference [14], James Zanotti for RBC/UKQCD has announced that a simulation at a second finer lattice spacing $(a \simeq 0.09 \mathrm{fm})$ is being performed and corresponding results for the vector form factor will be presented soon. Two other improvements on the existing calculation are being implemented, namely a simulation at a second value of the strange quark mass (the value of $m_{s}$ firstly simulated by RBC/UKQCD [9] was about $15 \%$ heavier than the physical strange quark) and the use of twisted boundary conditions. The latters, applied to 


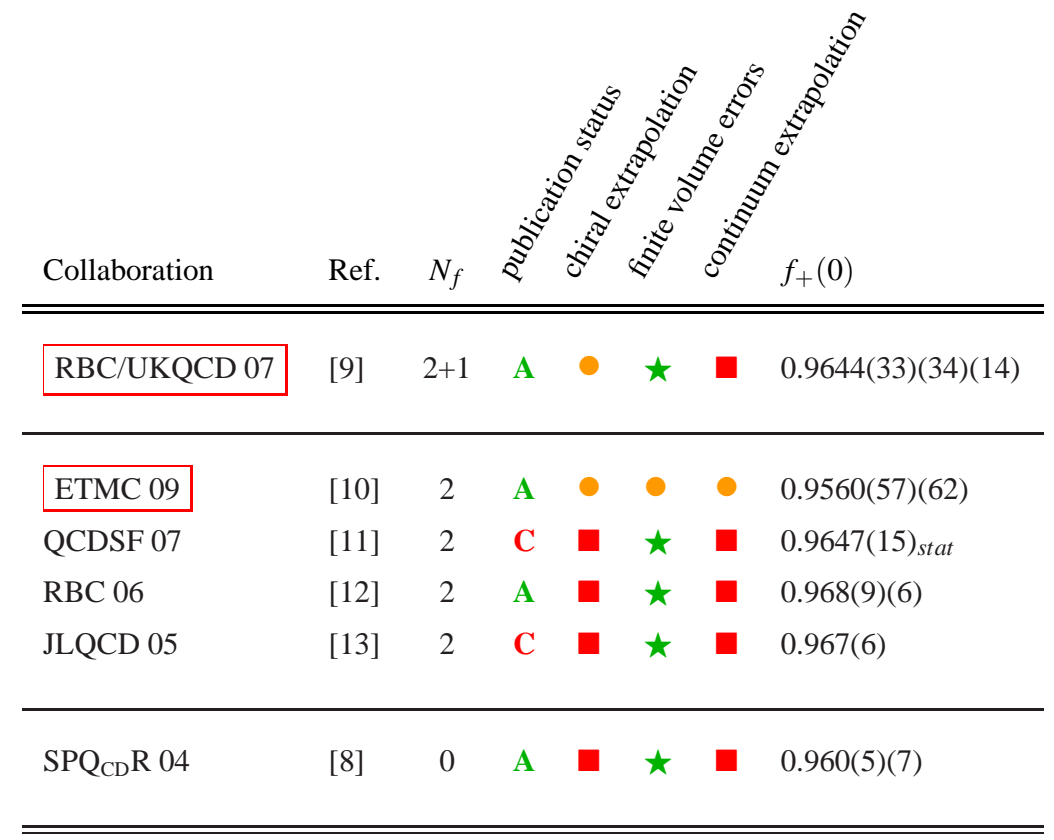

Table 2: Colour code rating in the FLAG style and summary of lattice results for $f_{+}(0)$. The results discussed at this conference are framed.

\begin{tabular}{|c|c|c|c|c|c|c|}
\hline Collaboration & Ref. & $N_{f}$ & action & $a / \mathrm{fm}$ & $\left(L m_{\pi}\right)^{\min }$ & $m_{\pi} / \mathrm{MeV}$ \\
\hline RBC/UKQCD 07 & [9] & $2+1$ & DWF & 0.11 & 4.6 & $\gtrsim 330$ \\
\hline ETMC 09 & [10] & 2 & $\max . \mathrm{tmQCD}$ & $\gtrsim 0.07$ & 3.7 & $\gtrsim 260$ \\
\hline QCDSF 07 & [11] & 2 & clover (NP) & 0.08 & 5.4 & $\gtrsim 590$ \\
\hline RBC 06 & {$[12]$} & 2 & DWF & 0.12 & 4.7 & $\gtrsim 490$ \\
\hline JLQCD 05 & {$[13]$} & 2 & clover (NP) & 0.09 & 4.9 & $\gtrsim 550$ \\
\hline $\mathrm{SPQ}_{\mathrm{CD}} \mathrm{R} 04$ & [8] & 0 & clover (NP) & 0.07 & 4.6 & $\gtrsim 500$ \\
\hline
\end{tabular}

Table 3: Parameters of the simulations listed in table 2.

valence quark fields $[16,17,18]$, allow to simulate very close or directly at $q^{2}=0$, thus removing the model dependence associated with the momentum extrapolation.

The ETMC calculation [10] of $f_{+}(0)$ has been presented at this conference by Silvano Simula [15]. It is based on the set of $N_{f}=2$ simulations performed by ETMC with twisted mass fermions at maximal twist. Finite size effects on the form factor have been estimated by simulating on two different volumes, whereas discretization errors have been evaluated by performing, for a single value of the light quark mass, calculations at three different lattice spacing $(a \simeq 0.07,0.09,0.10$ $\mathrm{fm}) .{ }^{1}$ In order to get results close to $q^{2}=0$, twisted boundary conditions on the valence quark fields have been implemented. Both pole and quadratic fits have been considered to interpolate to

\footnotetext{
${ }^{1}$ The results on the coarsest lattice have been analysed after the conference and are discussed in the proceedings [15].
} 
$q^{2}=0$. In the main simulation on the lattice with $a \simeq 0.09 \mathrm{fm}, 6$ different values of the light quark masses have been simulated, with the lightest pion mass being $m_{\pi} \simeq 260 \mathrm{MeV}$.

The relatively large number of light quark masses simulated by ETMC allows to achieve a good control over the chiral extrapolation, which represents one of the main source of systematic uncertainty in the lattice evaluation of $f_{+}(0)$. The chiral extrapolation has been performed using both SU(3) and, for the first time, SU(2) ChPT. The use of SU(2) ChPT applied to kaon observables [19] has been mainly advocated, in the context of lattice calculations, by RBC/UKQCD [20]. For a general discussion on the applicability of the different versions of ChPT to lattice results see the plenary talk by Enno Scholz at this conference [22]. For the vector form factor $f_{+}(0)$, the SU(2) chiral expansion has been derived at the NLO by Flynn and Sachrajda [21]. ETMC finds that the convergence of the $\mathrm{SU}(2)$ expansion for $f_{+}(0)$ is indeed very good, and contributions beyond NLO are small both at the physical point and in the region of simulated lattice data. Instead, both ETMC and RBC/UKQCD observe that the contribution beyond the NLO in the SU(3) chiral expansion, i.e. the quantity $\Delta f=f_{+}(0)-\left(1+f_{2}\right)$, is large and of the same size of the NLO contribution $f_{2}$. Nevertheless, the analyses based on SU(2) and SU(3) ChPT performed by ETMC leads eventually to consistent results, and their difference is included in the final estimate of the systematic error.

The ETMC calculation is performed with $N_{f}=2$ dynamical flavours. The effects due to the strange quark loops are exactly accounted for, in the calculation, up to the NLO in the SU(3) chiral expansion. This has been possible, because the NLO term in the chiral expansion, $f_{2}$, can be precisely computed (in terms of pion and kaon masses only) for the theories with $N_{f}=0$ [8], $N_{f}=$ 2 [23] and $N_{f}=2+1$ [6] dynamical quarks. Thus, the only uncertainty due to the quenching of the strange quark in the ETMC calculation concerns the NNLO contribution $\Delta f$. For this quantity, the relative uncertainty has been estimated by ETMC to be of the order of $13 \%$ which turns out to be of the same size of the entire difference $(\Delta f)^{N f=2}-(\Delta f)^{N f=0}$, evaluated using the quenched estimate of $\Delta f$ of ref. [8]. This difference is most likely an overestimate of the true error affecting $\Delta f$ in the $N_{f}=2$ calculation, i.e. $(\Delta f)^{\text {phys. }}-(\Delta f)^{N f=2}$.

In order to quote a lattice average for $f_{+}(0)$, I will take into account both the RBC/UKQCD and ETMC results, i.e.

$$
\begin{array}{ll}
f_{+}(0)=0.964(3)(4) & {\left[N_{f}=2+1, \mathrm{RBC} / \mathrm{UKQCD} 07\right]} \\
f_{+}(0)=0.956(6)(6) & {\left[N_{f}=2, \text { ETMC 09 }\right] .}
\end{array}
$$

These are the only results which are obtained with relatively light pion masses and do not get therefore in table 2 a red square for the chiral extrapolation. The RBC/UKQCD calculation has a red square assigned for the continuum extrapolation, but this source of error is subdominant in the calculation (even if the estimated error of $4 \%$ were doubled, the impact on the final result would be small). On the other hand, except for the partial quenching, the $N_{f}=2$ calculation by ETMC has at present a better control over the other systematic uncertainties with respect to the $N_{f}=2+1$ calculation by RBC/UKQCD (3 lattice spacings rather than 1, 6 pion masses rather than 4, both $\mathrm{SU}(3)$ and $\mathrm{SU}(2)$ chiral extrapolations). Since the uncertainty due to the quenching of the strange quark has been accounted for in the ETMC result, I quote as the best estimate of $f_{+}(0)$ the average of the two results in eq. (3.7), obtaining

$$
f_{+}(0)=0.962(3)(4)
$$




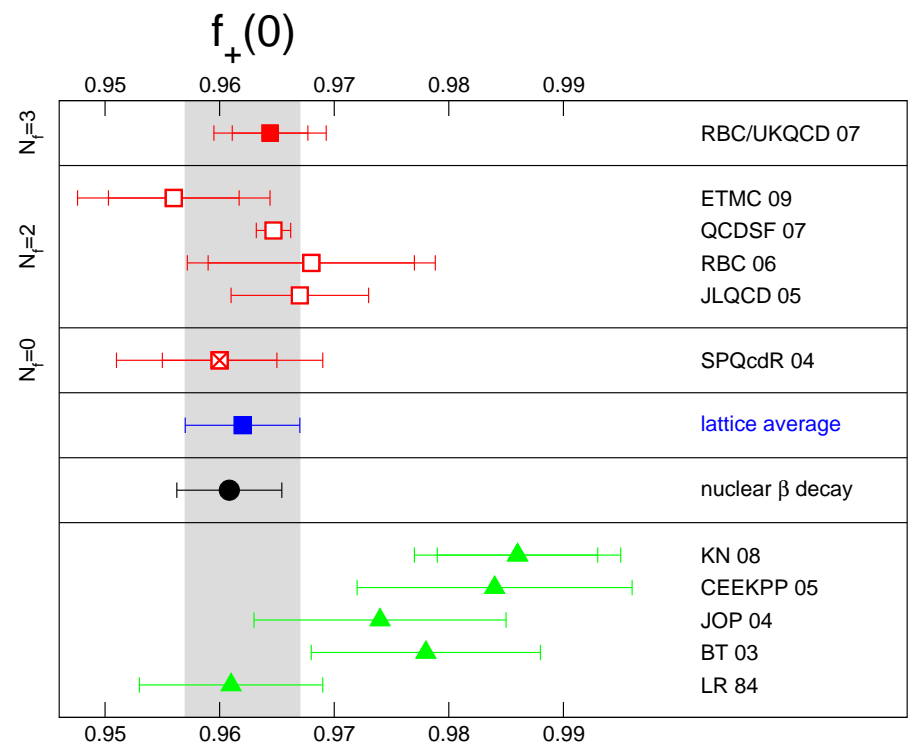

Figure 1: Summary of results for the vector form factor $f_{+}(0)$ of kaon semileptonic decays. Lattice results, as obtained from simulations with $N_{f}=0,2,3$ dynamical quarks, are shown in the upper side of the plot by red squares. The results of analytical model calculations [7],[26]-[29] are shown in the lower side of the plot by green triangles. The black circle represents the lattice-independent estimate of $f_{+}(0)$ given in eq. (3.5), which is only valid in the Standard Model. The blue square and the grey band show the average of lattice results for $f_{+}(0)$ derived in eq. (3.8). This plot has been produced by FLAG and updated for the lattice conference.

The first error in eq. (3.8) is statistical, and it is evaluated in the standard way assuming gaussian statistical uncertainties. The second error is systematic, and it has been taken to be equal to the systematic error quoted by RBC/UKQCD. ${ }^{2}$

The lattice average (3.8) is in very good agreement with the lattice-independent estimate quoted in eq. (3.5), based on CKM unitarity and the determination of $V_{u d}$ from nuclear $\beta$-decays. Combining the lattice determination (3.8) with the experimental result of eq. (3.1), one obtains the estimate

$$
\left|V_{u s}\right|_{K_{\ell 3}}=0.2252(13)
$$

in good agreement with the unitarity determination in eq. (3.4).

A summary of lattice results for $f_{+}(0)$ is shown in fig. 1 , which is an updated version for the lattice conference of a plot produced by FLAG [1]. The blue square and grey band in the plot

\footnotetext{
${ }^{2}$ While this contribution was almost finished to be written, an updated estimate of the form factor has been presented by RBC/UKQCD [24], which reads

$$
f_{+}(0)=0.9599(34)\left({ }_{-43}^{+31}\right)(14) .
$$

The new analysis includes the simulation at a second value of the strange quark mass and twisted boundary conditions to simulate directly at $q^{2}=0$, as anticipated at this conference. With respect to ref. [9], the change in the central value, as well as the second error in eq. (3.9), are due to the uncertainty in the chiral extrapolation, which was not considered in ref. [9]. It has been evaluated by varying in the SU(3) chiral expansion the value of the LO low-energy constant $f$ in the range 100-131 MeV (ETMC finds that the effect of a similar change in its chiral fit is smaller than the error already assigned to the chiral extrapolation [25]). Since the updated result (3.9) is in very good agreement with the lattice average given in eq. (3.8), I find unnecessary to change this average.
} 
represent the lattice average (3.8). The lattice-independent estimate (3.5) of $f_{+}(0)$, obtained using the unitarity determination of $V_{u s}$, is also shown for comparison. In the lower side of the plot, we collect the quark model result of Leutwyler and Roos (LR 84) [7] together with the results of more recent analytical model calculations [26]-[29]. These latter determinations of $f_{+}(0)$ turn out to be larger than both the value predicted by lattice QCD and the result implied by CKM unitarity.

\subsection{Leptonic kaon decays: $\mathbf{f}_{\mathbf{K}} / \mathbf{f}_{\pi}$}

A detailed review of lattice results for the pion and kaon decay constants has been given by Enno Scholz in his plenary talk at this conference [22]. For this reason, in this talk I will only summarize the main features of the new calculations and derive a lattice average for the ratio $f_{K} / f_{\pi}$, which is the relevant hadronic input parameter for the determination of the Cabibbo angle.

A list of unquenched lattice results for $f_{K} / f_{\pi}$ is collected in table 4 (based on the FLAG work), with the colour code rating in the FLAG style assigned for each calculation. The relevant simulation parameters for these calculations are given in table 5.

A first look at tables 4 and 5 is sufficient to reveal the large number of unquenched predictions for $f_{K} / f_{\pi}$ obtained by the various lattice collaborations in the last one or two years. For the purpose of this conference, the results which are new or have been updated in the last year are framed in the tables. Moreover, several lattice predictions for $f_{K} / f_{\pi}$ in table 4 are rated without red squares, indicating that all sources of systematic uncertainties in these calculations are kept sufficiently well under control.

At this conference, an update of the MILC analysis has been presented by Urs Heller [34]. This analysis is based on SU(3) (rooted) staggered ChPT and includes, as a new feature, the $\mathrm{N}^{2} \mathrm{LO}$ continuum chiral logs. The chiral fits are based on results obtained with the "fine", "super-fine" and "ultra-fine" MILC ensembles and are performed in two stages. The first one only includes the lowest quark masses, and it is used to determine the LO and NLO low energy constants. Once these constants are fixed, then the higher order contributions, namely the complete $\mathrm{N}^{2} \mathrm{LO}$ contribution together with $\mathrm{N}^{3} \mathrm{LO}$ and $\mathrm{N}^{4} \mathrm{LO}$ analytic terms, are determined through a global fit over all quark masses.

The RBC-UKQCD analysis of $f_{K} / f_{\pi}$, presented at this conference by Bob Mawhinney [33], is based on $\mathrm{SU}(2)$ chiral fits. While previous results by the collaboration were obtained at a single value of the lattice spacing $\left(a^{-1}=1.72 \mathrm{GeV}\right)$, the new analysis includes data from a second ensemble with a finer lattice $\left(a^{-1}=2.32 \mathrm{GeV}\right)$, which also includes lighter dynamical quarks. In addition, on the coarse lattice more configurations have been generated, by almost doubling the statistics from earlier works.

An accurate prediction for $f_{K} / f_{\pi}$ has been also presented by the BMW collaboration [31, 32], based on their extensive simulation performed at three values of the lattice spacing, large volumes and simulated pion masses reaching down about $190 \mathrm{MeV}$. The chiral extrapolation is performed testing three different assumptions for the quark mass dependence: ChPT, either SU(3) or SU(2), or a simple polynomial extrapolation. With respect to the preliminary determination presented in a poster by Alberto Ramos at this conference, the final result for $f_{K} / f_{\pi}$ given in $[31,32]$ and quoted in table 4 has a reduced systematic uncertainty.

The JLQCD/TWQCD collaboration has presented a preliminary determination of the pion and kaon decay constants [36] based on a simulation with $N_{f}=2+1$ dynamical overlap fermions, at 


\begin{tabular}{|c|c|c|c|c|c|c|c|}
\hline Collaboration & Ref. & $N_{f}$ & $\sqrt[2]{2}$ & $\vec{c}$ & $\Leftrightarrow$ & $0^{5}$ & $f_{K} / f_{\pi}$ \\
\hline \begin{tabular}{|l|} 
ALVdW 09 \\
\end{tabular} & {$[30]$} & $2+1$ & $\mathrm{C}$ & - & - & $\bullet$ & $1.192(12)(16)$ \\
\hline \begin{tabular}{|l|} 
BMW 09 \\
\end{tabular} & {$[31,32]$} & $2+1$ & $\mathbf{P}$ & $\star$ & $\star$ & $\star$ & $1.192(7)(6)$ \\
\hline RBC/UKQCD 09 & {$[33]$} & $2+1$ & $\mathbf{C}$ & - & $\star$ & - & $1.225(12)(14)$ \\
\hline MILC 09b & [34] & $2+1$ & $\mathbf{A}$ & $\star$ & $\star$ & $\star$ & $1.198(2)\left({ }_{-8}^{+6}\right)$ \\
\hline MILC 09a & {$[35]$} & $2+1$ & $\mathbf{A}$ & $\star$ & $\star$ & $\star$ & $1.197(3)\left({ }_{-13}^{+6}\right)$ \\
\hline JLQCD/TWQCD 09 & {$[36]$} & $2+1$ & $\mathbf{C}$ & $\bullet$ & $\mathbf{\square}$ & - & $1.210(12)_{\text {stat }}$ \\
\hline PACS-CS 08 & {$[37]$} & $2+1$ & $\mathbf{A}$ & $\star$ & - & $\mathbf{\square}$ & $1.189(20)$ \\
\hline HPQCD/UKQCD 07 & {$[38]$} & $2+1$ & $\mathbf{A}$ & $\star$ & - & $\star$ & $1.189(2)(7)$ \\
\hline RBC/UKQCD 08 & {$[20]$} & $2+1$ & $\mathbf{A}$ & $\bullet$ & $\star$ & $\mathbf{\square}$ & $1.205(18)(62)$ \\
\hline NPLQCD 06 & [39] & $2+1$ & $\mathbf{A}$ & - & $\mathbf{\square}$ & 口 & $1.218(2)\left({ }_{-24}^{+11}\right)$ \\
\hline MILC 04 & [40] & $2+1$ & $\mathbf{A}$ & $\star$ & $\bullet$ & $\bullet$ & $1.210(4)(13)$ \\
\hline \begin{tabular}{|l} 
ETMC 09 \\
\end{tabular} & [41] & 2 & $\mathbf{A}$ & $\bullet$ & $\bullet$ & $\star$ & $1.210(6)(15)(9)$ \\
\hline ETMC 07 & {$[42]$} & 2 & $\mathbf{A}$ & - & - & $\mathbf{\square}$ & $1.227(9)(24)$ \\
\hline QCDSF/UKQCD 07 & {$[43]$} & 2 & $\mathrm{C}$ & $\bullet$ & $\star$ & $\bullet$ & $1.21(3)$ \\
\hline
\end{tabular}

Table 4: Colour code rating in the FLAG style and summary of unquenched lattice results for $f_{K} / f_{\pi}$. The new results, obtained in the last year, are framed.

\begin{tabular}{|c|c|c|c|c|c|c|}
\hline Collaboration & Ref. & $N_{f}$ & action & $a / \mathrm{fm}$ & $\left(L m_{\pi}\right)^{\min }$ & $m_{\pi} / \mathrm{MeV}$ \\
\hline ALVdW 09 & {$[30]$} & $2+1$ & $\mathrm{KS}_{\mathrm{MILC}} / \mathrm{DWF}$ & $\gtrsim 0.09$ & 3.8 & $\gtrsim 300$ \\
\hline BMW 09 & {$[31,32]$} & $2+1$ & impr. Wilson & $\gtrsim 0.07$ & 4.0 & $\gtrsim 190$ \\
\hline RBC/UKQCD 09 & {$[33]$} & $2+1$ & DWF & $\gtrsim 0.08$ & 4.0 & $\gtrsim 290$ \\
\hline MILC 09b & [34] & $2+1$ & $\mathrm{KS}_{\mathrm{MILC}}^{\mathrm{MILC}}$ & $\gtrsim 0.045$ & 4.0 & $\gtrsim 230$ \\
\hline MILC 09a & {$[35]$} & $2+1$ & $\mathrm{KS}_{\mathrm{MILC}}^{\mathrm{MILC}}$ & $\gtrsim 0.045$ & 3.8 & $\gtrsim 230$ \\
\hline JLQCD/TWQCD 09 & {$[36]$} & $2+1$ & Overlap & 0.10 & 2.8 & $\gtrsim 340$ \\
\hline PACS-CS 08 & {$[37]$} & $2+1$ & clover (NP) & 0.09 & 2.3 & $\gtrsim 160$ \\
\hline HPQCD/UKQCD 07 & {$[38]$} & $2+1$ & $\mathrm{KS}_{\mathrm{MILC}}^{\mathrm{HISQ}}$ & $\gtrsim 0.09$ & 3.8 & $\gtrsim 250$ \\
\hline RBC/UKQCD 08 & {$[20]$} & $2+1$ & DWF & 0.11 & 4.6 & $\gtrsim 330$ \\
\hline NPLQCD 06 & [39] & $2+1$ & $\mathrm{KS}_{\mathrm{MILC}} / \mathrm{DWF}$ & 0.13 & 3.7 & $\gtrsim 290$ \\
\hline MILC 04 & {$[40]$} & $2+1$ & $\mathrm{KS}_{\text {MILC }}^{\mathrm{MILC}}$ & $\gtrsim 0.09$ & 3.8 & $\gtrsim 250$ \\
\hline \begin{tabular}{|l|} 
ETMC 09 \\
\end{tabular} & [41] & 2 & max. tmQCD & $\gtrsim 0.07$ & 3.2 & $\gtrsim 260$ \\
\hline ETMC 07 & {$[42]$} & 2 & $\max . \mathrm{tmQCD}$ & 0.09 & 3.2 & $\gtrsim 300$ \\
\hline QCDSF/UKQCD 07 & {$[43]$} & 2 & clover (NP) & $\gtrsim 0.06$ & 4.2 & $\gtrsim 300$ \\
\hline
\end{tabular}

Table 5: Parameters of the simulations listed in table 4. 
five different up and down quark masses. While the chosen approach is definitively a benchmark for lattice QCD, since the overlap formulation preserves an exact chiral symmetry at finite lattice spacing, the simulation performed by JLQCD/TWQCD relies yet on a single value of the lattice spacing $(a \simeq 0.10 \mathrm{fm})$ and a rather small lattice size $\left(\left(L m_{\pi}\right)^{\mathrm{min}}<3\right)$, two features which imply the red squares in the FLAG stye rating of table 4.

Among the other $N_{f}=2+1$ determinations of $f_{K} / f_{\pi}$ collected in table 4 and not presented at this conference, the ALVdW 09 and HPQCD/UKQCD 08 results are also free of red tags and will be thus included in the determination of the final lattice average.

A determination of $f_{K} / f_{\pi}$ with $N_{f}=2$ dynamical quarks, in which all sources of systematic uncertainties are kept under control (i.e. no red squares in the colour code rating) has been recently obtained by the ETM collaboration [41], using maximally twisted mass fermions. The comparison with the most precise $N_{f}=2+1$ calculations suggests that the quenching effect of the strange quark in the determination of $f_{K} / f_{\pi}$ is currently negligible, within the reached lattice accuracy. Therefore, I will include this result in the determination of the final lattice average (in this case, given the large number of independent determinations of $f_{K} / f_{\pi}$ which are free of red squares in table 4, the inclusion of the ETM 09 result has a marginal impact). I then obtain

$$
f_{K} / f_{\pi}=1.196(1)(10),
$$

where the first error is statistical, evaluated assuming gaussian statistical uncertainties, and the second error is systematic. For the latter, I'm quoting an uncertainty which is of the same size of the typical systematic uncertainty estimated for the most precise lattice determinations listed in table 4.

The average (3.11) can be translated into a determination of the kaon decay constant, $f_{K}$, by using $f_{\pi}=130.41(3)(20) \mathrm{MeV}$ [44], as determined from the measurement of the leptonic pion decay rate and $V_{u d}$ from eq. (3.3). In this way one gets

$$
f_{K}=156.0 \pm 1.3 \mathrm{MeV} \text {. }
$$

The lattice average (3.11) is also in very good agreement with the lattice-independent estimate of $f_{K} / f_{\pi}$ quoted in eq. (3.5), based on CKM unitarity and the determination of $V_{u d}$ from nuclear $\beta$-decays. By combining the lattice prediction (3.11) with the experimental result of eq. (3.1) and with $V_{u d}$ from nuclear $\beta$-decays, one obtains the estimate

$$
\left|V_{u s}\right|_{K_{\ell 2}}=0.2249(19)
$$

This in good agreement with both the determination (3.10) from $K_{\ell 3}$ decays and with the unitarity determination in eq. (3.4).

A summary of lattice results for $f_{K} / f_{\pi}$ is shown in fig. 1, which is an updated version for the lattice conference of a plot produced by FLAG [1]. The blue square and grey band in the plot represents the lattice average (3.11). The lattice-independent estimate (3.5) of $f_{K} / f_{\pi}$ is also shown for comparison. 


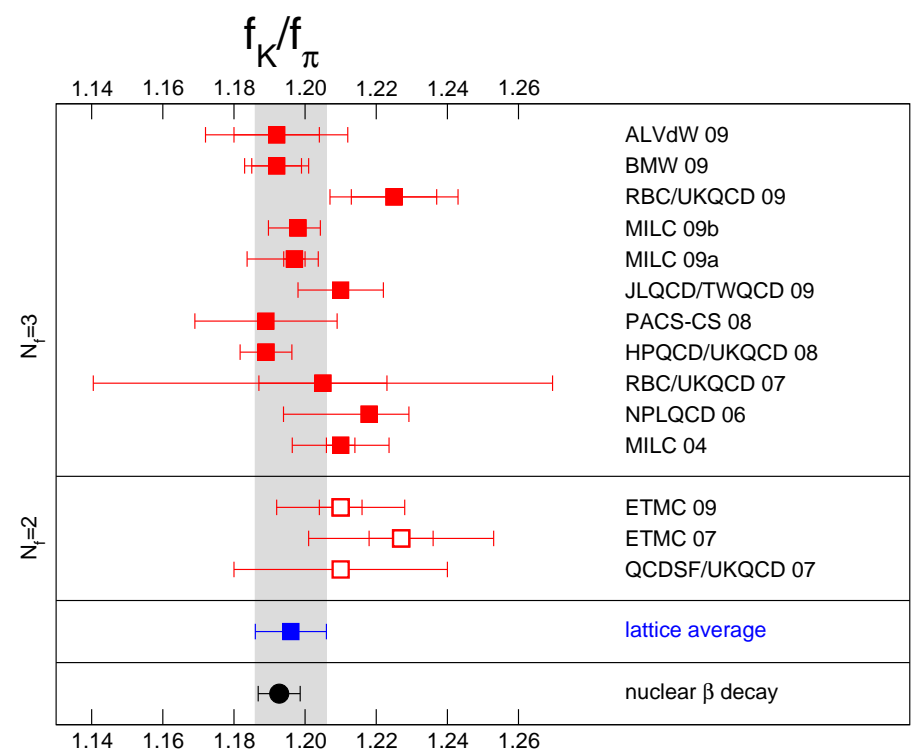

Figure 2: Summary of unquenched lattice results for the ratio of decay constants $f_{K} / f_{\pi}$ as obtained from simulations with $N_{f}=2$ and $N_{f}=3$ dynamical quarks (red squares). The black circle represents the latticeindependent estimate of $f_{K} / f_{\pi}$ given in eq. (3.5), which is valid only in the Standard Model. The blue square and the grey band show the average of the lattice results for $f_{K} / f_{\pi}$ derived in eq. (3.11). This plot has been produced by FLAG and updated for the lattice conference.

\section{4. $\mathbf{K}^{\mathbf{0}}-\overline{\mathbf{K}}^{\mathbf{0}}$ mixing: $\mathbf{B}_{\mathbf{K}}$}

The accuracy in the lattice determination of the kaon bag parameter, $B_{K}$, has remarkably improved over the last few years. This progress is well illustrated by the following sample of lattice averages presented at the lattice conferences:

$$
\begin{array}{lcl}
\text { Lattice'96 } & \hat{B}_{K}=0.90 \pm 0.03 \pm 0.15 & \text { S. Sharpe, [45] } \\
\text { Lattice'00 } & \hat{B}_{K}=0.86 \pm 0.06 \pm 0.14 & \text { L. Lellouch, [46] } \\
\text { Lattice'05 } & \hat{B}_{K}=0.79 \pm 0.04 \pm 0.08 & \text { C. Dawson, [47] } \\
\text { Lattice'08 } & \hat{B}_{K}=0.723 \pm 0.037 & \text { L. Lellouch, [48] }
\end{array}
$$

where $\hat{B}_{K}$ is the renormalization group invariant definition (for $N_{f}=3$ ) of the bag parameter. The second error quoted in eqs. (4.1)-(4.3) was an estimate of the quenched error. This uncertainty has limited the accuracy of the lattice calculations of $B_{K}$ for a long time. It has started to decrease when the first unquenched estimates have been performed in the last few years, and in the average of $B_{K}$ quoted at the last two lattice conferences it was definitively removed. Nevertheless, all unquenched determinations of $B_{K}$ available until last year were obtained at a fixed (and rather large) lattice spacing. Thus, a quantitative estimate of discretization effects affecting these calculations, which could have been not negligible according to the experience gathered in the quenched approximation, was not available yet.

The list of unquenched lattice results for $B_{K}$ available today is collected in table 6 , with the colour code rating in the FLAG style assigned for each calculation. The relevant simulation param- 


\begin{tabular}{|c|c|c|c|c|c|c|c|c|c|c|}
\hline Collaboration & Ref. & $N_{f}$ & $\sqrt[3]{2}$ & $0^{\circ}$ & ci & ज्ञ & E & $s$ & $B_{\mathrm{K}}^{\overline{\mathrm{MS}}}(2 \mathrm{GeV})$ & $\hat{B}_{\mathrm{K}}$ \\
\hline ALVdW 09 & [49] & $2+1$ & $\mathbf{A}$ & $\bullet$ & $\star$ & - & $\star$ & - & $0.527(6)(20)$ & $0.724(8)(28)$ \\
\hline RBC/UKQCD 09 & {$[50]$} & $2+1$ & C & $\bullet$ & $\bullet$ & $\star$ & $\star$ & $\bullet$ & $0.537(6)(18)$ & $0.738(8)(25)$ \\
\hline \begin{tabular}{|l|} 
SBW 09 \\
\end{tabular} & {$[51]-[54]$} & $2+1$ & $\mathrm{C}$ & $\star$ & $\star$ & $\mathbf{\square}$ & $\mathbf{\square}$ & - & $0.512(14)(34)$ & $0.701(19)(47)$ \\
\hline RBC/UKQCD 07 & {$[55,20]$} & $2+1$ & $\mathbf{A}$ & 口 & $\bullet$ & $\star$ & $\star$ & $\bullet$ & $0.524(10)(28)$ & $0.720(13)(37)$ \\
\hline HPQCD/UKQCD 06 & {$[56]$} & $2+1$ & $\mathbf{A}$ & 口 & $\bullet$ & $\star$ & $\square$ & ○ & $0.618(18)(135)$ & $0.83(18)$ \\
\hline \begin{tabular}{|l|} 
ETMC 09 \\
\end{tabular} & {$[57]$} & 2 & $\mathrm{C}$ & $\star$ & - & - & $\star$ & - & $0.518(21)(21)$ & $0.730(30)(30)$ \\
\hline JLQCD 08 & {$[58]$} & 2 & $\mathbf{A}$ & $\mathbf{\square}$ & $\bullet$ & $\mathbf{\square}$ & $\star$ & $\bullet$ & $0.537(4)(40)$ & $0.758(6)(71)$ \\
\hline RBC 04 & [59] & 2 & $\mathbf{A}$ & $\mathbf{\square}$ & $\mathbf{\square}$ & $\mathbf{a}^{\dagger}$ & $\star$ & - & $0.495(18)$ & $0.699(25)$ \\
\hline UKQCD 04 & {$[60]$} & 2 & $\mathbf{A}$ & $\mathbf{\square}$ & $\square$ & $\mathbf{a}^{\dagger}$ & 口 & - & $0.49(13)$ & $0.69(18)$ \\
\hline
\end{tabular}

Table 6: Colour code rating in the FLAG style and summary of unquenched lattice results for $B_{K}$. The symbol $\square^{\dagger}$ means that these results have been obtained at $\left(M_{\pi} L\right)_{\min }>4$ in a lattice box with a spatial extension $L<2 \mathrm{fm}$. The new results obtained in the last year are framed.

\begin{tabular}{|c|c|c|c|c|c|c|c|}
\hline Collaboration & Ref. & $N_{f}$ & action & $a / \mathrm{fm}$ & $\left(L m_{\pi}\right)^{\min }$ & $m_{\pi} / \mathrm{MeV}$ & Ren. \\
\hline \begin{tabular}{|l|} 
ALVdW 09 \\
\end{tabular} & [49] & $2+1$ & $\mathrm{KS}_{\mathrm{MILC}} / \mathrm{DWF}$ & $\gtrsim 0.09$ & 3.5 & $\gtrsim 230$ & RI \\
\hline RBC/UKQCD 09 & {$[50]$} & $2+1$ & DWF & $\gtrsim 0.08$ & 4.0 & $\gtrsim 290$ & RI \\
\hline SBW 09 & {$[51]-[54]$} & $2+1$ & $\mathrm{KS}_{\text {MILC }}^{\mathrm{HYP}}$ & $\gtrsim 0.06$ & 2.5 & $\gtrsim 200$ & PT1 $\ell$ \\
\hline RBC/UKQCD 07 & {$[55,20]$} & $2+1$ & DWF & 0.11 & 4.6 & $\gtrsim 330$ & RI \\
\hline HPQCD/UKQCD 06 & {$[56]$} & $2+1$ & $\mathrm{KS}_{\text {MILC }}^{\mathrm{MILC}}$ & 0.12 & 4.6 & $\gtrsim 360$ & PT1 $\ell$ \\
\hline \begin{tabular}{|l|} 
ETMC 09 \\
\end{tabular} & [57] & 2 & $\mathrm{tmQCD} / \mathrm{OS}$ & $\gtrsim 0.07$ & 3.2 & $\gtrsim 260$ & RI \\
\hline JLQCD 08 & {$[58]$} & 2 & overlap & 0.12 & 2.8 & $\gtrsim 290$ & RI \\
\hline $\mathrm{RBC} 04$ & [59] & 2 & DWF & 0.12 & 4.6 & $\gtrsim 490$ & RI \\
\hline UKQCD 04 & {$[60]$} & 2 & clover(NP) & 0.10 & 6.2 & $\gtrsim 740$ & PT1 $\ell$ \\
\hline
\end{tabular}

Table 7: Parameters of the simulations listed in table 6.

eters for these calculations are given in table 7.

The novelty this year are a number of new calculations, namely ALVdW 09 [49], RBC/ UKQCD 09 [50], SBW 09 [51]-[54] and ETMC 09 [57], which are framed in table 6 for better illustration. At variance with the previous unquenched calculations, they all involve an extrapolation to the continuum limit for $B_{K}$, based on two (ALVdW 09, RBC/UKQCD 09) or three (SBW 09, 
ETMC 09) values of the lattice spacing.

While the RBC/UKQCD 09, SBW 09 and ETMC 09 results are still preliminary, having been only presented in the proceedings of this conference, the ALVdW 09 calculation is already published [49]. It uses a mixed action setup, with domain wall fermions valence quarks over the $N_{f}=2+1$ staggered gauge field configurations produced by MILC ("fine" and "coarse" lattices). The choice of domain wall fermions for the valence allows a straightforward implementation of the RI-MOM method in order to non-perturbatively renormalize the four-fermion operator relevant for $B_{K}$ (in the previous determination of $B_{K}$ with staggered fermions by HPQCD/UKQCD [56], the one-loop perturbative determination of the renormalization constant turned out to be affected by an uncomfortably large systematic uncertainty). With domain wall fermions, due to the residual breaking of chiral symmetry which is allowed by the finite extension of the lattice in the fifth dimension, the $B_{K}$ operator has a small mixing with operators of wrong chirality (but not with those of incorrect taste). The final result for the bag parameter is obtained after performing a combined chiral and continuum extrapolation based on NLO SU(3) "mixed action" ChPT [61, 62], with the inclusion of some analytic $\mathrm{N}^{2} \mathrm{LO}$ contribution. The final accuracy quoted for $B_{K}$ is about $4 \%$, where the dominant source of uncertainty is represented by the determination of the renormalization constant.

The new result for $B_{K}$ obtained by RBC/UKQCD, which updates the first precise unquenched calculation of ref. [55, 20], has been presented by Chris Kelly at this conference [50]. The main improvement with respect to the previous calculation is the use of the second finer lattice ensemble simulated by RBC/UKQCD, which allows to perform a combined SU(2)-chiral and continuum extrapolation of $B_{K}$. This, in turn, has permitted a substantial reduction of the systematic error ( $3.5 \%$ ), since in the previous calculation discretization effects represented the main source of uncertainty. The new calculation also makes use of reweighting in the strange sea sector, with a corresponding interpolation in the valence sector, to reach the physical strange quark mass. Renormalization of the four-fermion operator is performed non-perturbatively using the RI-MOM approach generalized to various non-exceptional momentum renormalization conditions (for a detailed discussion of this approach see the plenary talk by Yasumichi Aoki at this conference [63]).

The new SBW 09 calculation [51]-[54] (where the acronym indicates the Seoul, Brookhaven and Washington institutions) uses a mixed action setup, with HYP-smeared staggered valence fermions, which are effective at reducing taste-breaking effects, and the asqtad staggered sea quarks, i.e. the MILC ensembles. Results are obtained at three values of the lattice spacing, namely the "coarse", "fine" and "super-fine" MILC ensembles. The chiral extrapolation is performed by using the proper either SU(3) or SU(2) mixed action staggered ChPT at NLO, with or without adding an analytical $\mathrm{N}^{2} \mathrm{LO}$ term. The $\mathrm{SU}(2)$ result, which is the one quoted in table 6 , leads eventually to a smaller error. Since the minimum value of $m_{\pi} L$ in the simulation is about 2.5 , this calculation gets a red square in table 6 for finite volume errors. These errors, however, have been explicitly investigated by the collaboration and they are found to be subdominant for $B_{K}$, smaller than $1 \%$ for the $\mathrm{SU}(2)$ analysis. The main uncertainty in the calculation comes from the evaluation of the renormalization constant, which is performed in one-loop perturbation theory. With respect to the older staggered calculation of ref. [56], this uncertainty is now significantly reduced, mainly because of the use of the "fine" and "super-fine" MILC ensembles for which the error coming from the truncation of the perturbative series, of order $\alpha_{s}(1 / a)^{2}$, is expected to be smaller. Nevertheless 
it still dominates the systematic uncertainty. The collaboration plans to reduce this uncertainty in various way. One is to work on a yet finer lattice, another is to use two-loop matching and, finally, by using the RI-MOM non-perturbative method. This latter approach, which looks the most promising, has been already successfully applied with staggered fermions to the renormalization of bilinear quark operators, as discussed by Andrew Lytle at this conference [64].

The fourth new result for $B_{K}$ has been presented at this conference by the ETM collaboration [57]. This calculation also uses a mixed action setup, with $N_{f}=2$ maximally twisted sea quarks and a suitable Osterwalder-Seiler variant of the twisted mass action for the valence quarks. This setup simultaneously guarantees the absence of mixing with operators of wrong chirality, which is usually present with Wilson-like fermions, and automatic $\mathscr{O}(a)$-improvement of $B_{K}$ [65]. The calculation is performed at three values of the lattice spacing $(a \simeq 0.07,0.09,0.10 \mathrm{fm})$ and the physical value of $B_{K}$ is eventually reached through a combined $\mathrm{SU}(2)$-chiral and continuum extrapolation. Renormalization of the four fermion operator is performed non-perturbatively with the RI-MOM method. Discretization effects in the evaluation of the renormalization constant, which starts at $\mathscr{O}\left(g^{2} a^{2}\right)$, are further reduced by subtracting from the RI-MOM four-fermion Green function the leading contribution, explicitly evaluated in $\mathscr{O}\left(a^{2}\right)$ one-loop lattice perturbation theory, as illustrated by Fotos Stylianou in a poster at this conference [66].

In order to derive the final lattice average for $B_{K}$, I consider the three results which are free of red squares in table 6 , namely:

$$
\begin{array}{ll}
\hat{B}_{K}=0.724(8)(28) & {\left[N_{f}=2+1, \text { ALVdW 09 }\right]} \\
\hat{B}_{K}=0.738(8)(25) & {\left[N_{f}=2+1, \text { RBC } / \text { UKQCD 09 }\right]} \\
\hat{B}_{K}=0.730(30)(30) & {\left[N_{f}=2, \text { ETMC 09 }\right] .}
\end{array}
$$

The agreement among the above determinations, as well as with the new SBW 09 determination [51]-[54], is remarkable. It is also worth noticing that the result obtained with $N_{f}=2$ dynamical quarks lies in between the two $N_{f}=2+1$ determinations, showing that also for $B_{K}$ the effect of quenching the strange quark is not visible, within the accuracy currently reached by lattice calculations. For this reason, I average together the three results in eq. (4.5) and quote as the best lattice estimate of $\hat{B}_{K}$ the value

$$
\hat{B}_{K}=0.731(7)(35) .
$$

While the statistical error is evaluated assuming gaussian statistical uncertainties, the systematic uncertainty quoted in eq. (4.6) is slightly increased with respect to the one quoted by the individual calculations, to account for the preliminary status of two out of the three calculations on which the final average of $\hat{B}_{K}$ is based. A summary of the unquenched results for $\hat{B}_{K}$ is also presented in fig. 3, which is an updated version for this conference of a plot produced by FLAG [1]. The average value of $\hat{B}_{K}$, given in eq. (4.6), is shown in the plot with a blue square and a grey band.

The $\varepsilon_{K}$ parameter which controls the amount of indirect CP violation in $K^{0}-\bar{K}^{0}$ mixing, and whose theoretical determination relies on $B_{K}$, plays a relevant role in the unitarity triangle analysis, both within and beyond the Standard Model. Since in the Standard Model the analysis is largely overconstrained, it can be also used to extract the values of the relevant hadronic parameters, including $B_{K}$ [67]. The latest determination obtained in this way by the UTfit collaboration, which 


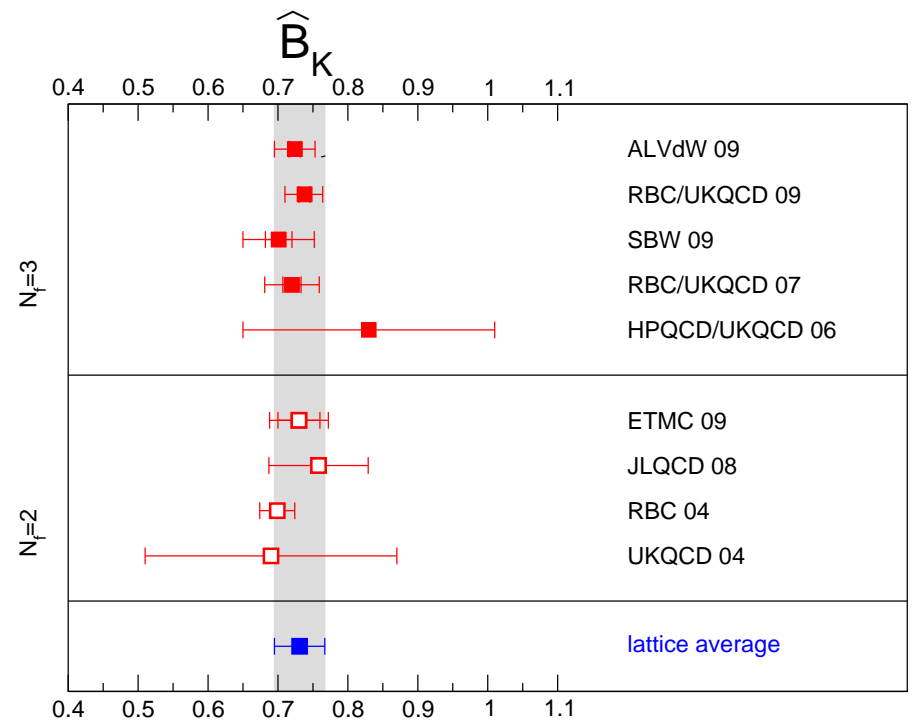

Figure 3: Summary of unquenched lattice results for the RGI parameter $\hat{B}_{K}$ as obtained from simulations with $N_{f}=2$ and $N_{f}=3$ dynamical quarks (red squares). The blue square and the grey band show the average of the lattice results derived in eq. (4.6). This plot has been produced by FLAG and updated for the lattice conference.

is therefore only valid in the Standard Model, is

$$
\left(\hat{B}_{K}\right)_{\mathrm{UTfit}}^{\mathrm{SM}}=0.87(8)
$$

This estimate also takes into account the long distance contributions to both the absorptive and the dispersive part of the $\Delta S=2$ effective Hamiltonian evaluated in refs. [68, 69] and summarized in the multiplicative factor $k_{\varepsilon}=0.94 \pm 0.02$. The Standard Model prediction of $B_{K}$ from the unitarity triangle analysis, given in eq. (4.7), shows a tension with the direct lattice determination (4.6), at the level of $1.6 \sigma$. Whether such a tension should persist, with the increasing precision of both lattice calculations and of the unitarity triangle analysis, it could become a clear signal of physics beyond the Standard Model.

In new physics models, like for instance the supersymmetric extensions of the Standard Model, the theoretical expression of $\varepsilon_{K}$ depends in general on the complete basis of eight $\Delta S=2$ fourfermion operators [70]. Due to parity conservation in the strong interactions, only five of these operators have independent matrix elements. The knowledge of these matrix elements is then crucial in order to derive reliable predictions for $\varepsilon_{K}$ in the context of specific new physics models. Lattice calculations for the complete basis of $\Delta S=2$ four-fermion operators have been only performed so far in the quenched approximation [71, 72, 73], and the results turn out to be in poor agreement among each other. Very preliminary results for the full basis of $\Delta S=2$ matrix elements have been presented at this conference by ETMC [57]. Given their high phenomenological interest, it would be helpful if such a calculation were also address by other collaborations using different lattice approaches. 


\section{Non leptonic kaon decays}

While not much time (and space) is left to discuss the lattice studies of non leptonic kaon decays, I cannot conclude this talk without at least mentioning the extraordinary effort which is being put forward by the RBC/UKQCD collaboration in addressing this issue. The results obtained last year by the collaboration have shown that the attractive approach which uses ChPT to relate the $K \rightarrow \pi \pi$ matrix element of interest to the simpler matrix elements of $K \rightarrow \pi$ and $K \rightarrow 0$ transitions is affected, in the kaon mass region, by significantly large chiral corrections. This is an intrinsic uncertainty, which cannot be avoided. Therefore, the direct calculation of $K \rightarrow \pi \pi$ matrix elements on the lattice must be addressed.

At this conference, three talks have been dedicated to this topic by RBC/UKQCD [74, 75, 76]. The main indication is that, while a substantial computational efforts will be required in order to obtain a reasonably accurate estimate (i.e. at the level of 10-20\%), the direct calculation of both the $A_{0}$ and $A_{2}$ complex amplitudes is however feasible. The required theoretical and numerical tools include, in particular, the use of chiral fermions, non-perturbative RI-MOM renormalization and finite volume methods.

An exhaustive description of this topic would require a dedicated talk by itself. For that, I would like to refer the reader to the excellent review given by Norman Christ at the KAON'09 conference [77].

\section{Summary and outlook}

The number of large unquenched lattice simulations which are being applied to the study kaon physics is rapidly increasing. This effort has allowed to achieve in the lattice determination of some (relatively simple) kaon physics observables an unprecedented accuracy. In this talk, I concentrated most of the attention in reviewing the lattice results for three of these parameters, namely the vector semileptonic form factor $f_{+}(0)$, the ratio of decay constants $f_{K} / f_{\pi}$ and the kaon bag parameter $B_{K}$. Lattice averages for these parameters have been given in eqs. (3.8), (3.11) and (4.6). Lattice studies of non leptonic kaon decays are significantly more challenging. Nevertheless, important progress has been achieved also in this field, and first, reliable results for both the $\Delta I=1 / 2$ rule and $\varepsilon^{\prime} / \varepsilon$ are expected to produced in two ore three years.

\section{Acknowledgement}

I would like to thank many colleagues who helped me in preparing this talk, by providing useful information and/or anticipating to me their results: Claude Bernard, Norman Christ, Urs Heller, Weonjong Lee, Laurent Lellouch, Chris Kelly, Matthew Lightman, Bob Mawhinney, Alberto Ramos, Steve Sharpe, Ruth Van de Water, James Zanotti and all my colleagues in the ETM collaboration.

A special thank goes to the members of the FLAG working group, Gilberto Colangelo, Stephan Dürr, Andreas Jüttner, Laurent Lellouch, Heiri Leutwyler, Silvia Necco, Chris Sachrajda, Silvano Simula, Tassos Vladikas, Urs Wenger and Hartmut Wittig. Much of the material that I have presented in this talk, including tables and plots, has been prepared in collaboration with them. 
Finally I would like to thank the organizers of Lattice 2009 for inviting me to give this review and for planning a very enjoyable meeting.

\section{References}

[1] G. Colangelo et al. [FLAG Working Group of FLAVIANET], "Review of lattice results concerning low energy particle physics”, in preparation. See also G. Colangelo, talk given at EuroFlavour 2009, http://www.ba.infn.it/indico/conferenceDisplay p py?conf Id=4 9

[2] R. Baron et al., arXiv:0911.5244 [hep-lat].

[3] M. Antonelli et al. [FlaviaNet Working Group on Kaon Decays], arXiv:0801.1817 [hep-ph].

[4] J. C. Hardy and I. S. Towner, Phys. Rev. C 79 (2009) 055502 [arXiv:0812.1202 [nucl-ex]].

[5] M. Ademollo and R. Gatto, Phys. Rev. Lett. 13 (1964) 264.

[6] J. Gasser and H. Leutwyler, Nucl. Phys. B 250 (1985) 517.

[7] H. Leutwyler and M. Roos, Z. Phys. C 25 (1984) 91.

[8] D. Becirevic et al., Nucl. Phys. B 705 (2005) 339 [arXiv:hep-ph/0403217].

[9] P. A. Boyle et al., Phys. Rev. Lett. 100 (2008) 141601 [arXiv:0710.5136 [hep-lat]].

[10] V. Lubicz, F. Mescia, S. Simula and C. Tarantino [ETM Collaboration], Phys. Rev. D 80 (2009) 111502 [arXiv:0906.4728 [hep-lat]].

[11] D. Brommel et al. [QCDSF collaboration], PoS LAT2007 (2007) 364 [arXiv:0710.2100 [hep-lat]].

[12] C. Dawson, T. Izubuchi, T. Kaneko, S. Sasaki and A. Soni, Phys. Rev. D 74 (2006) 114502 [arXiv:hep-ph/0607162].

[13] N. Tsutsui et al. [JLQCD Collaboration], PoS LAT2005 (2006) 357 [arXiv:hep-lat/0510068].

[14] RBC and UKQCD Collaborations, talk given by J. Zanotti at this conference.

[15] S. Di Vita, B. Haas, F. Mescia, V. Lubicz, S. Simula and C. Tarantino [ETM Collaboration], PoS LAT2009 (2009) 257 [arXiv:0910.4845 [hep-lat]].

[16] P. A. Boyle, J. M. Flynn, A. Juttner, C. T. Sachrajda and J. M. Zanotti, JHEP 0705 (2007) 016 [arXiv:hep-lat/0703005].

[17] D. Guadagnoli, F. Mescia and S. Simula, Phys. Rev. D 73 (2006) 114504 [arXiv:hep-lat/0512020].

[18] C. T. Sachrajda and G. Villadoro, Phys. Lett. B 609 (2005) 73 [arXiv:hep-lat/0411033].

[19] A. Roessl, Nucl. Phys. B 555 (1999) 507 [arXiv:hep-ph/9904230].

[20] C. Allton et al. [RBC-UKQCD Collaboration], Phys. Rev. D 78 (2008) 114509 [arXiv:0804.0473 [hep-lat]].

[21] J. M. Flynn and C. T. Sachrajda [RBC and UKQCD Collaborations], Nucl. Phys. B 812 (2009) 64 [arXiv:0809.1229 [hep-ph]].

[22] E. E. Scholz, PoS LAT2009 (2009) 005 arXiv:0911.2191 [hep-lat].

[23] D. Becirevic, G. Martinelli and G. Villadoro, Phys. Lett. B 633 (2006) 84 [arXiv:hep-lat/0508013].

[24] P. A. Boyle et al., arXiv:1004.0886 [Unknown].

[25] S. Simula, private communication. 
[26] A. Kastner and H. Neufeld, Eur. Phys. J. C 57 (2008) 541 [arXiv:0805.2222 [hep-ph]].

[27] V. Cirigliano, G. Ecker, M. Eidemuller, R. Kaiser, A. Pich and J. Portoles, JHEP 0504 (2005) 006 [arXiv:hep-ph/0503108].

[28] M. Jamin, J. A. Oller and A. Pich, JHEP 0402 (2004) 047 [arXiv:hep-ph/0401080].

[29] J. Bijnens and P. Talavera, Nucl. Phys. B 669 (2003) 341 [arXiv:hep-ph/0303103].

[30] C. Aubin, J. Laiho and R. S. Van de Water, talk given by J. Laiho at Chiral Dynamics 2009, Bern, Switzerland, July 6-10, 2009. http://www.chiral09.unibe.ch/

[31] A. Ramos and f. M. collaboration, PoS LAT2009 (2009) 259 [arXiv:1002.1665 [hep-lat]].

[32] S. Durr et al., arXiv:1001.4692 [hep-lat].

[33] R. Mawhinney [RBC Collaboration and UKQCD Collaboration], PoS LAT2009 (2009) 081 [arXiv:0910.3194 [hep-lat]]. CI,LAT2009,081;

[34] A. Bazavov et al. [The MILC Collaboration], PoS LAT2009 (2009) 079 [arXiv:0910.3618 [hep-lat]].

[35] A. Bazavov et al., arXiv:0903.3598 [hep-lat].

[36] J. Noaki et al. [JLQCD and TWQCD Collaborations], PoS LAT2009 (2009) 096 [arXiv:0910.5532 [hep-lat]].

[37] S. Aoki et al. [PACS-CS Collaboration], Phys. Rev. D 79 (2009) 034503 [arXiv:0807.1661 [hep-lat]].

[38] E. Follana, C. T. H. Davies, G. P. Lepage and J. Shigemitsu [HPQCD Collaboration and UKQCD Collaboration], Phys. Rev. Lett. 100 (2008) 062002 [arXiv:0706.1726 [hep-lat]].

[39] S. R. Beane, P. F. Bedaque, K. Orginos and M. J. Savage, Phys. Rev. D 75 (2007) 094501 [arXiv:hep-lat/0606023].

[40] C. Aubin et al. [MILC Collaboration], Phys. Rev. D 70 (2004) 114501 [arXiv:hep-lat/0407028].

[41] B. Blossier et al., JHEP 0907 (2009) 043 [arXiv:0904.0954 [hep-lat]].

[42] B. Blossier et al. [ETM Collaboration], JHEP 0804 (2008) 020 [arXiv:0709.4574 [hep-lat]].

[43] G. Schierholz, talk given at Lattice 2007, Regensburg, Germany, http://www.physik.uni-regensburg.de/lat07/hevea/schierholz.pdf

[44] J. L. Rosner and S. Stone, arXiv:1002.1655 [hep-ex].

[45] S. R. Sharpe, Nucl. Phys. Proc. Suppl. 53 (1997) 181 [arXiv:hep-lat/9609029].

[46] L. Lellouch, Nucl. Phys. Proc. Suppl. 94 (2001) 142 [arXiv:hep-lat/0011088].

[47] C. Dawson, PoS LAT2005, 007 (2006).

[48] L. Lellouch, PoS LATTICE2008 (2009) 015 [arXiv:0902.4545 [hep-lat]].

[49] C. Aubin, J. Laiho and R. S. Van de Water, Phys. Rev. D 81 (2010) 014507 [arXiv:0905.3947 [hep-lat]].

[50] C. Kelly, P. A. Boyle and C. T. Sachrajda [RBC Collaboration and UKQCD Collaboration], PoS LAT2009 (2009) 087 [arXiv:0911.1309 [hep-lat]].

[51] T. Bae et al., PoS LAT2009 (2009) 261 [arXiv:0910.5576 [hep-lat]].

[52] H. J. Kim et al., PoS LAT2009 (2009) 262 [arXiv:0910.5573 [hep-lat]].

[53] B. Yoon et al., PoS LAT2009 (2009) 263 [arXiv:0910.5581 [hep-lat]]. 
[54] J. Kim et al., PoS LAT2009 (2009) 264 [arXiv:0910.5583 [hep-lat]].

[55] D. J. Antonio et al. [RBC Collaboration and UKQCD Collaboration], Phys. Rev. Lett. 100 (2008) 032001 [arXiv:hep-ph/0702042].

[56] E. Gamiz, S. Collins, C. T. H. Davies, G. P. Lepage, J. Shigemitsu and M. Wingate [HPQCD Collaboration and UKQCD Collaboration], Phys. Rev. D 73 (2006) 114502 [arXiv:hep-lat/0603023].

[57] V. Bertone et al. [ETM Collaboration], PoS LAT2009 (2009) 258 [arXiv:0910.4838 [hep-lat]].

[58] S. Aoki et al. [JLQCD Collaboration], Phys. Rev. D 77 (2008) 094503 [arXiv:0801.4186 [hep-lat]].

[59] Y. Aoki et al., Phys. Rev. D 72 (2005) 114505 [arXiv:hep-lat/0411006].

[60] J. M. Flynn, F. Mescia and A. S. B. Tariq [UKQCD Collaboration], JHEP 0411 (2004) 049 [arXiv:hep-lat/0406013].

[61] O. Bar, C. Bernard, G. Rupak and N. Shoresh, Phys. Rev. D 72 (2005) 054502 [arXiv:hep-lat/0503009].

[62] C. Aubin, J. Laiho and R. S. Van de Water, Phys. Rev. D 75 (2007) 034502 [Erratum-ibid. D 79 (2009) 079904] [arXiv:hep-lat/0609009].

[63] Y. Aoki, plenary talk at this conference, http://rchep.pku.edu.cn/workshop/lattice09/Jul 2028 20TUE/Aoki.pdf

[64] A. T. Lytle, PoS LAT2009 (2009) 202 [arXiv:0910.3721 [hep-lat]].

[65] R. Frezzotti and G. C. Rossi, JHEP 0410 (2004) 070 [arXiv:hep-lat/0407002].

[66] M. Constantinou, V. Lubicz, H. Panagopoulos, A. Skouroupathis and F. Stylianou, arXiv:1001.1241 [hep-lat].

[67] M. Bona et al. [UTfit Collaboration], JHEP 0610 (2006) 081 [arXiv:hep-ph/0606167].

[68] A. J. Buras and D. Guadagnoli, Phys. Rev. D 78 (2008) 033005 [arXiv:0805.3887 [hep-ph]].

[69] A. J. Buras, D. Guadagnoli and G. Isidori, arXiv:1002.3612 [Unknown].

[70] M. Ciuchini et al., JHEP 9810 (1998) 008 [arXiv:hep-ph/9808328].

[71] A. Donini, V. Gimenez, L. Giusti and G. Martinelli, Phys. Lett. B 470 (1999) 233 [arXiv:hep-lat/9910017].

[72] R. Babich, N. Garron, C. Hoelbling, J. Howard, L. Lellouch and C. Rebbi, Phys. Rev. D 74 (2006) 073009 [arXiv:hep-lat/0605016].

[73] Y. Nakamura et al. [CP-PACS Collaboration], PoS LAT2006 (2006) 089 [arXiv:hep-lat/0610075].

[74] Q. Liu [RBC Collaboration and UKQCD Collaboration], arXiv:0910.2658 [hep-lat].

[75] M. Lightman and E. Goode [RBC Collaboration and UKQCD Collaboration], PoS LAT2009 (2009) 254 [arXiv:0912.1667 [Unknown]].

[76] C. Kim and N. H. Christ, PoS LAT2009 (2009) 255 [arXiv:0912.2936 [hep-lat]].

[77] N. H. Christ [RBC Collaboration and UKQCD Collaboration], arXiv:0912.2917 [hep-lat]. 